Supporting Information for

\title{
Chemical Stability and Transformation of Molybdenum Disulfide Nanosheets in Environmental Media
}

Ting-Wei Lee, Chia-Chi Chen, and Chiaying Chen*

Department of Environmental Engineering, National Chung Hsing University, Taichung City 402, Taiwan *Corresponding author: Tel: 886-4-22840441\#541. E-mail: chiayingchen@nchu.edu.tw

Number of pages: 18

Number of figures: 13

Number of tables: 1

Number of texts: 1

\section{Contents}

Text S1 Supplementary experimental section.

Table S1 Properties of NOM utilized in the present study.

Figure S1 C 1s XPS spectra of ALHA and SRNOM.

Figure S2 MoS2 concentrations determined by UV-vis absorbance and ICP-OES. Page S6

Figure S3 SEM image and Mo 3d XPS spectra of $\mathrm{MoS}_{2}$ bulk powder.

Page S7

Figure S4 S 2p XPS spectra of bulk $\mathrm{MoS}_{2}$ powder, $\mathrm{sMoS}_{2}$, and ceMoS2.

Page S8

Figure S5 Absorbance at $450 \mathrm{~nm}$ of NOM in the 123-day-aged process.

Page S9

Figure S6 Absorbance at $450 \mathrm{~nm}$ of $\mathrm{sMoS}_{2}$ in the 123-day-aged process.

Page S10

Figure S7 Raman spectra of $\mathrm{sMoS}_{2}$ sample.

Page S11

Figure S8 pH evolution of ceMoS 2 samples in the 123-day-aged process.

Page S12

Figure S9 Kinetic results of Mo-O formation and 1T (Mo-S) degradation.

Page S13

Figure S10 S 2p XPS spectra of ceMoS2 samples under dark ambient conditions.

Page S14

Figure S11 FTIR spectra of NOM and ceMoS2 samples.

Page S15

Figure S12 Absorbance at $450 \mathrm{~nm}$ of NOM used under sunlight exposure.

Page S16

Figure S13 Mott-Schottky plots of ALHA, SRNOM, and ceMoS2.

Page S17

References

Page S18 
Text S1. Supplementary experimental section

\section{Materials}

All reagents were of analytical grade and were used as received. Molybdenum (IV) sulfide (99\%), Pluronic F-127, Hydrochloric acid (37\%), Hexane ( $\geq 99 \%$ ), and Sodium hydroxide (98\%) were purchased from Sigma-Aldrich. n-Butyllithium solution (1.6 M in hexane) was from Rockwood Lithium. Suwannee River natural organic matter (SRNOM) and Aldrich humic acid (ALHA) were obtained from International Humic Substances Society and Sigma Aldrich, respectively. Aqueous solutions were prepared in water purified to $\geq 18.2 \mathrm{M} \Omega \cdot \mathrm{cm}$.

\section{Characterization}

A Zetasizer Nano ZS (Malvern Instrument, Worcestershire, U.K.) with a monochromatic coherent $633 \mathrm{~nm}$ He-Ne laser was used for measuring zeta potential and size distributions of the prepared materials in aqueous media. In zeta potential measurement, $\mathrm{pH}$ of the solution was adjusted to $3 \sim 9.5$ prior to analysis. The surface atomic percentage of chemical composition and distribution of functional groups of the prepared $\mathrm{MoS}_{2}$ suspensions were analyzed by X-ray photoelectron spectroscopy (XPS) (ULVAC-PHI, PHI 5000 VersaProbe/Scanning ESCA Microprobe). ceMoS 2 samples were deposited and air-drying on silicon substrates prior to XPS characterization. $\mathrm{sMoS}_{2}$ samples were concentrated and collected through filtering and subsequent oven-drying due to its weak XPS signals. The XPSPEAK41 software with Shirley background type was used, in which the background range was in accord with the fitting peaks of target elements (i.e., Mo and S). Functional groups of $\mathrm{MoS}_{2}$ nanosheet samples were characterized by Fourier transform infrared (FTIR) (Thermo Fisher Scientific, Nicolet iS5) spectrometer. The associations of NOM with $\mathrm{MoS}_{2}$ nanosheets were examined by Raman spectroscopy with $488 \mathrm{~nm}$ excitation laser (Tokyo Instrument, Nanofinder 30) with sample deposited on silicon substrate. The morphologies were obtained by high-resolution transmission electron microscopy (TEM) (JEOL JEM-1200) and scanning electron microscopy (SEM) (JEOL 
JSM-7800F). Electrochemical analysis was conducted in a three-electrode configuration comprised of an $\mathrm{Ag} / \mathrm{AgCl}$ electrode as the reference electrode, platinum wire as the counter electrode, and a glassy carbon electrode with ceMoS 2 and $\mathrm{sMoS}_{2}$ samples (1 mg of samples in a solution containing $0.7 \mathrm{~mL}$ deionized water and $50 \mu \mathrm{L}$ of $5 \mathrm{wt} \%$ Nafion aqueous solution) as the working electrode. The Mott-Schottky analysis was carried out to investigate the band structures of $\mathrm{MoS}_{2}$ nanosheet with samples deposited on a glassy carbon electrode. A $0.5 \mathrm{M} \mathrm{Na}_{2} \mathrm{SO}_{4}$ aqueous solution was used as the electrolyte. 
Table S1. Properties of NOM utilized in the present study.

\begin{tabular}{|c|c|c|c|c|c|c|c|c|c|c|c|}
\hline \multirow[t]{2}{*}{ NOM } & \multicolumn{5}{|c|}{ Elemental composition (wt \%) } & \multicolumn{6}{|c|}{ Functional group distributions (from assignment of ${ }^{13} \mathrm{C}$ NMR chemical shifts) } \\
\hline & $\mathbf{C}$ & $\mathbf{H}$ & $\mathbf{O}$ & $\mathbf{N}$ & $\mathbf{S}$ & $\begin{array}{c}\text { Aliphatic } \\
(0-60 \\
\text { ppm) }\end{array}$ & $\begin{array}{c}\text { Hetero- } \\
\text { aliphatic } \\
\text { (60-90 } \\
\text { ppm) }\end{array}$ & $\begin{array}{c}\text { Acetal } \\
(90-110 \\
\text { ppm) }\end{array}$ & $\begin{array}{c}\text { Aromatic } \\
(110-165 \\
\text { ppm) }\end{array}$ & $\begin{array}{c}\text { Carboxyl } \\
(165-190 \\
\text { ppm) }\end{array}$ & $\begin{array}{c}\text { Carbonyl } \\
(190-220 \\
\text { ppm) }\end{array}$ \\
\hline SRNOM $^{\mathbf{a}}$ & 52.5 & 4.2 & 42.7 & 1.1 & 0.7 & 27 & 15 & 7 & 23 & 20 & 8 \\
\hline ALHA $^{b}$ & 50.3 & 5.7 & 43.6 & 0.4 & N.D. & 13 & 19 & 34 & 34 & & \\
\hline
\end{tabular}

a : data from International Humic Substances Society (IHSS) ${ }^{1}$

b : data from Kang et al. ${ }^{2}$

\begin{tabular}{|c|c|c|c|c|c|c|c|c|}
\hline NOM & $\begin{array}{c}\text { SUVA254 }_{254} \\
\left(\mathrm{~L} \mathrm{mgC}^{-1} \mathrm{~m}^{-1}\right)^{\mathrm{c}}\end{array}$ & $\begin{array}{c}\text { SUVA280 } \\
\left(\mathrm{L} \mathrm{mgC}^{-1} \mathbf{~ m}^{-1}\right)^{\mathrm{c}} \\
\end{array}$ & $\begin{array}{c}\text { SUVA } 365_{365} \\
\left(\mathrm{~L} \mathrm{mgC}^{-1} \mathbf{~ m}^{-1}\right)^{\mathrm{c}}\end{array}$ & $\begin{array}{c}\text { SUVA465 }_{465} \\
\left(\mathrm{~L} \mathrm{mgC}^{-1} \mathbf{~ m}^{-1}\right)^{\mathrm{c}}\end{array}$ & $\begin{array}{c}\text { SUVA665 } \\
\left(\mathrm{L} \mathrm{mgC}^{-1} \mathbf{~ m}^{-1}\right)^{\mathrm{c}} \\
\end{array}$ & $\mathbf{E 2}: \mathbf{E} 3^{c}$ & E4 : E6 ${ }^{c}$ & $\begin{array}{c}\text { Aromaticity } \\
(\%)\end{array}$ \\
\hline SRNOM & 6.41 & 4.92 & 1.49 & 0.29 & N.D. & 4.30 & N.A. & $31^{\mathrm{d}}$ \\
\hline ALHA & 10.08 & 8.65 & 3.63 & 1.40 & 0.19 & 2.78 & 7.37 & $53^{\mathrm{e}}$ \\
\hline
\end{tabular}

c : measured in this study

d : data from Louie et al. ${ }^{3}$

e : data calculated from Chin et al. ${ }^{4}$

$\mathrm{E} 2: \mathrm{E} 3=\mathrm{SUVA}_{254} / \mathrm{SUVA}_{365}$

E4 : E6 $=$ SUVA $_{465} /$ SUVA $_{665}$

Aromaticity $=\varepsilon * 0.05+6.74$, where $\varepsilon=$ number-averaged molar absorptivity at $280 \mathrm{~nm}, \mathrm{R}^{2}=0.90 .{ }^{4}$ 

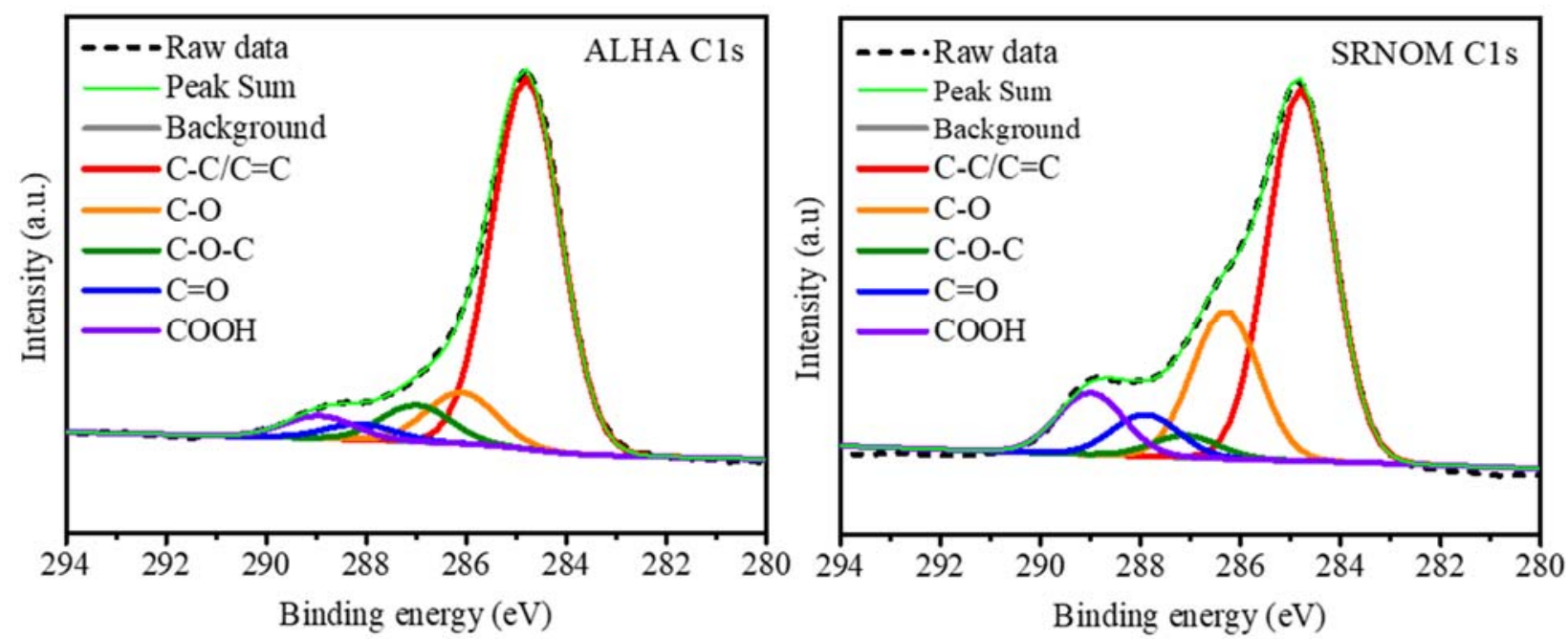

Figure S1. C 1s XPS spectra of ALHA and SRNOM used in the present study. 

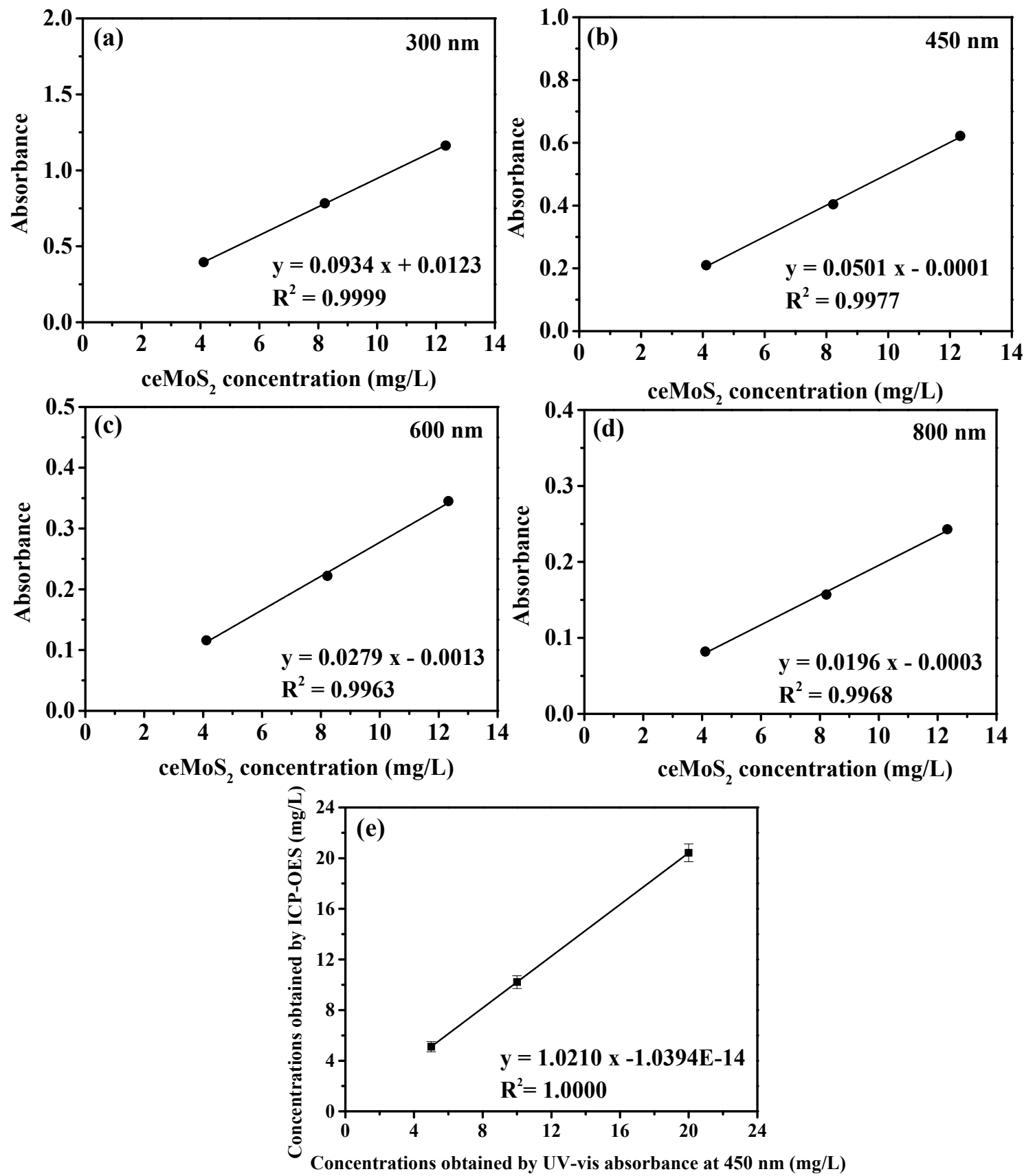

Figure S2. (a-d) Linear correlation of concentrations to optical absorbance at 300, 450, 600, 800 nm. (e) $\mathrm{MoS}_{2}$ concentrations determined by UV-vis absorbance at $450 \mathrm{~nm}$ and ICP-OES. The mass extinction coefficients were calculated to be $9340,5010,2790,1960 \mathrm{~L} \mathrm{~m}^{-1} \mathrm{~g}^{-1}$ at wavelengths of $300,450,600,800 \mathrm{~nm}$, respectively, in good agreement with the reported values. ${ }^{5,6}$ 

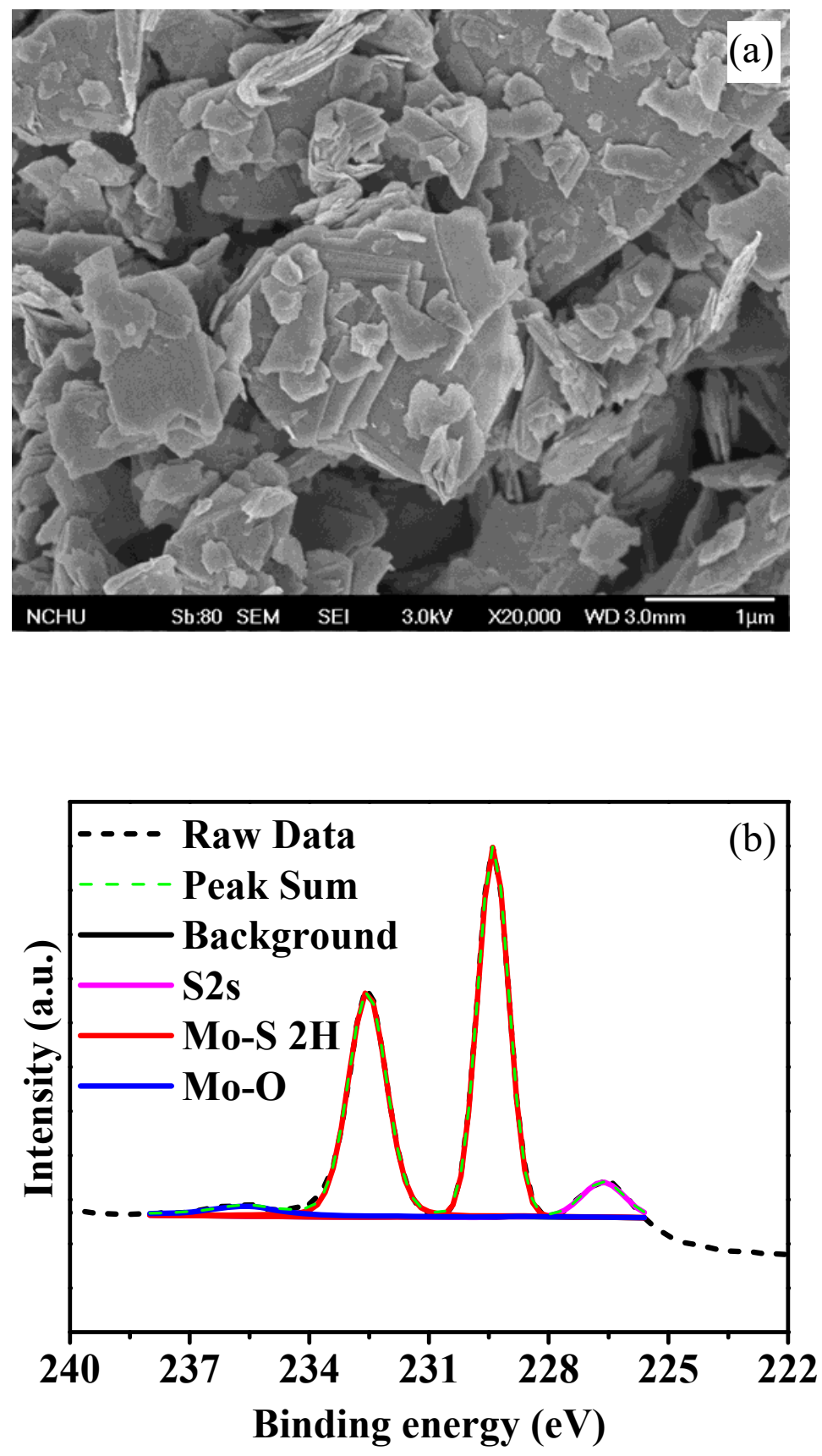

Figure S3. (a) SEM image of bulk $\mathrm{MoS}_{2}$ powder, showing a multilayered nature. (b) Mo 3d XPS spectra of $\mathrm{MoS}_{2}$ bulk powder, displaying a pure $2 \mathrm{H}$ phase. 

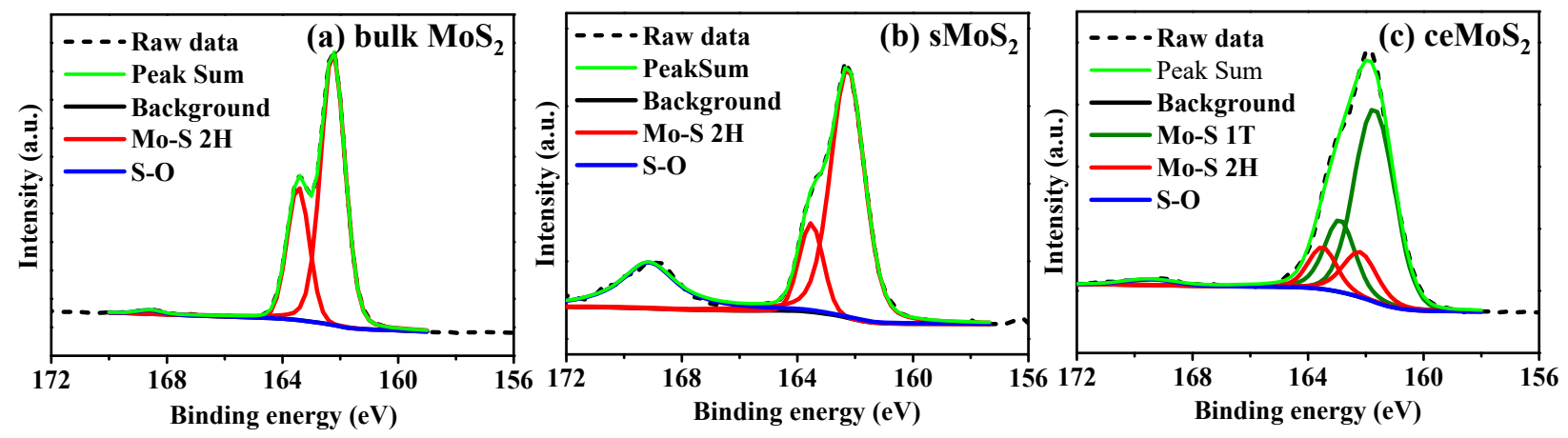

Figure S4. S 2p XPS spectra of (a) bulk $\mathrm{MoS}_{2}$ powder, (b) sMoS2, and (c) ceMoS2. 


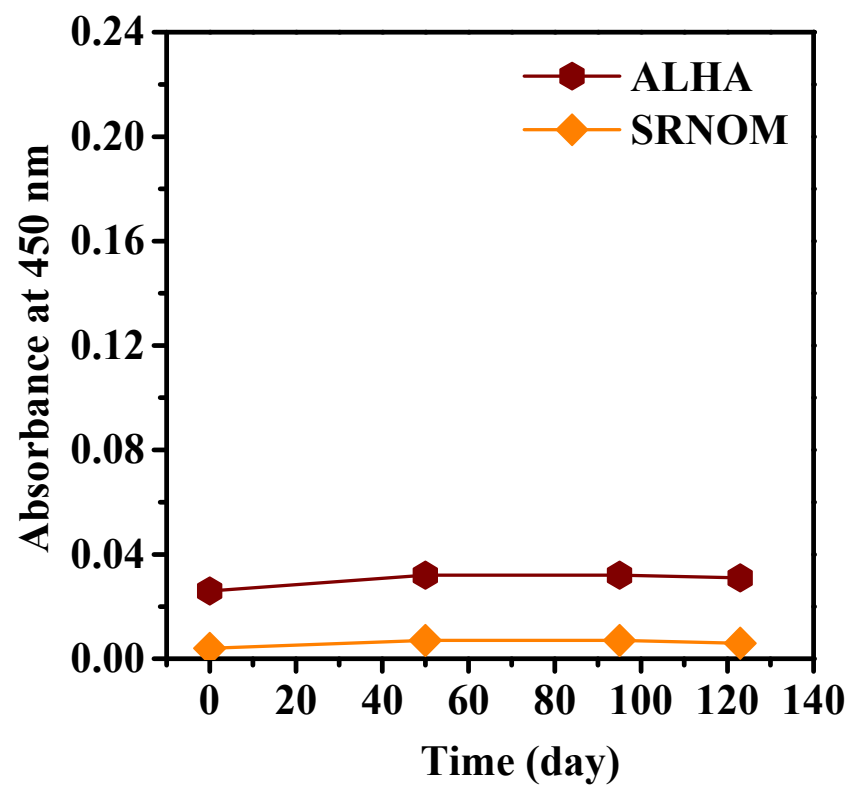

Figure S5. UV-vis absorbance at $450 \mathrm{~nm}$ of ALHA and SRNOM ( $8 \mathrm{mg} / \mathrm{L})$ in the 123-day-aged process under dark ambient conditions. 


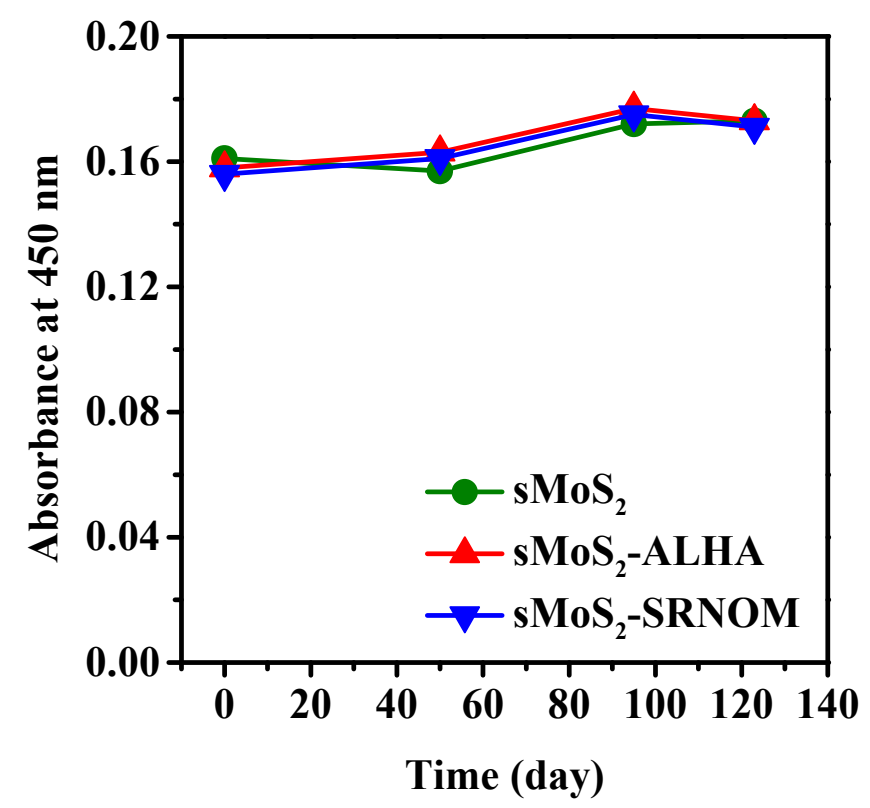

Figure S6. UV-vis absorbance at $450 \mathrm{~nm}$ of sMoS2 samples in the 123-day-aged process under dark ambient conditions, displaying a small $\triangle \mathrm{Abs}_{450}(<5 \%)$ of $\mathrm{sMoS}_{2}$, sMoS 2 -ALHA, and sMoS2-SRNOM. 


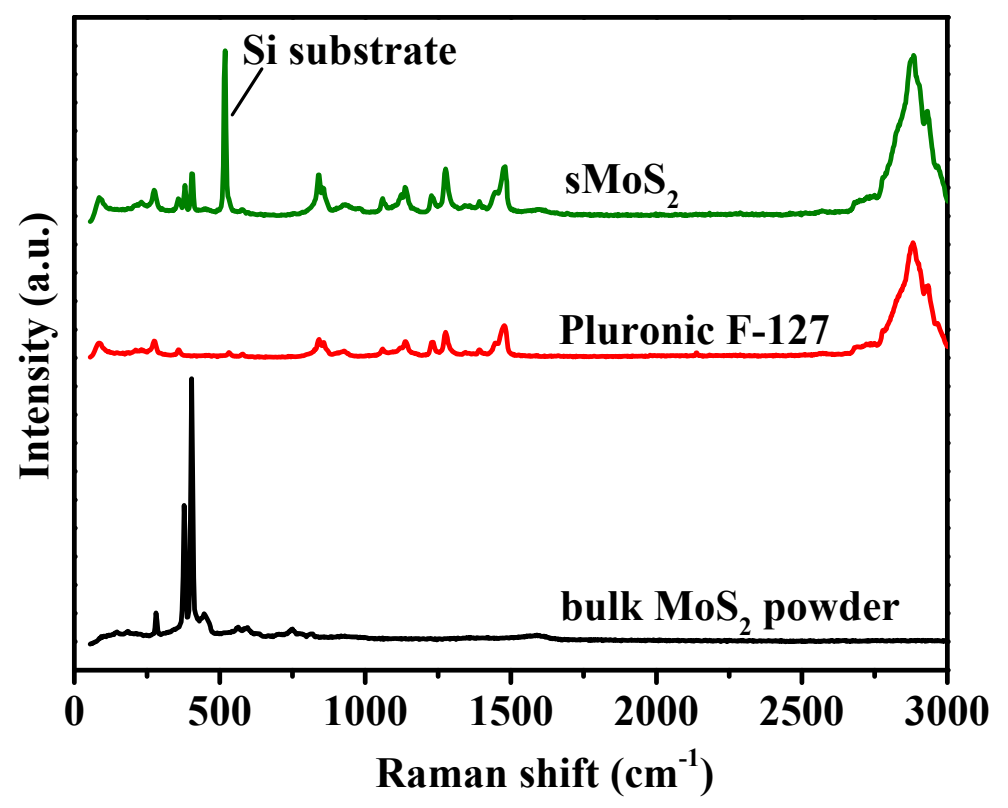

Figure S7. Raman spectra of 3-day-aged $\mathrm{sMoS}_{2}$ sample, as characteristic $\mathrm{CH}_{2}$ rocking vibration ( $\left.~ 845 \mathrm{~cm}^{-1}\right)$, C-O stretching vibration $\left(931 \mathrm{~cm}^{-1}, 1063 \mathrm{~cm}^{-1}, 1142 \mathrm{~cm}^{-1}\right), \mathrm{CH}_{2}$ twisting vibration (1233 $\left.\mathrm{cm}^{-1}\right), \mathrm{CH}_{2}$ wagging/C-C stretching vibration $\left(1395 \mathrm{~cm}^{-1}\right)$, and $\mathrm{CH}_{2}$ scissoring vibration $\left(1481 \mathrm{~cm}^{-1}\right)$ of Pluronics ${ }^{7}$ were observed in $\mathrm{sMoS}_{2}$. 


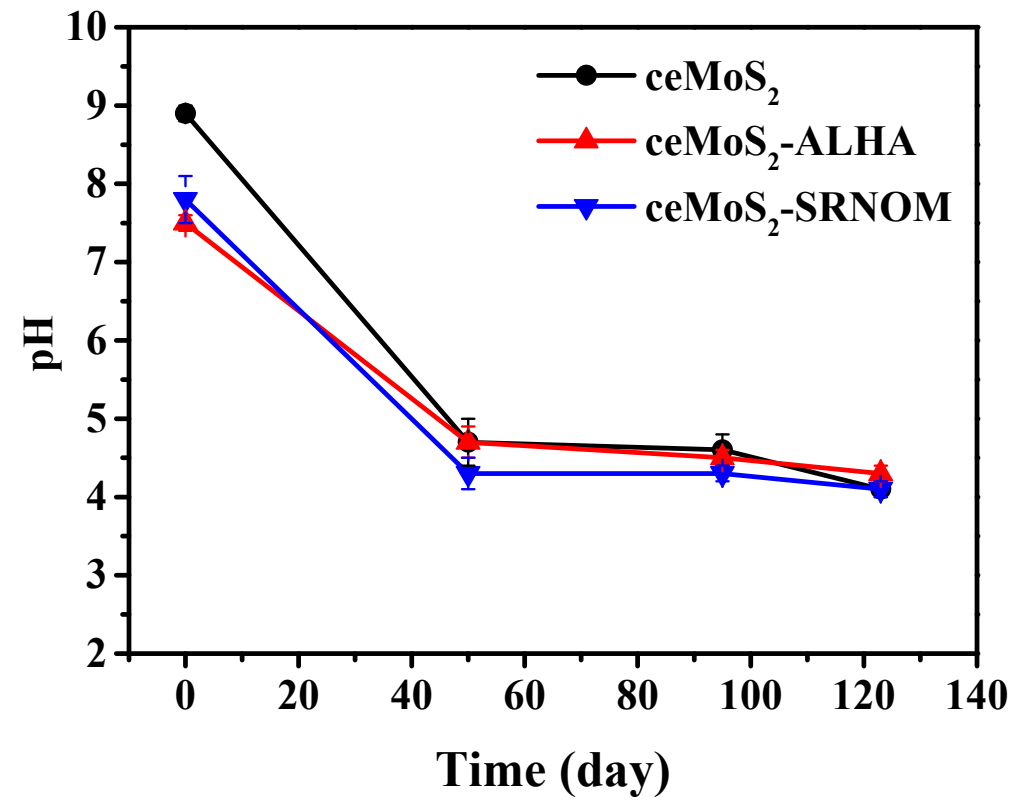

Figure S8. pH evolution of ceMoS 2 samples in the 123-day-aged process under dark ambient conditions. 


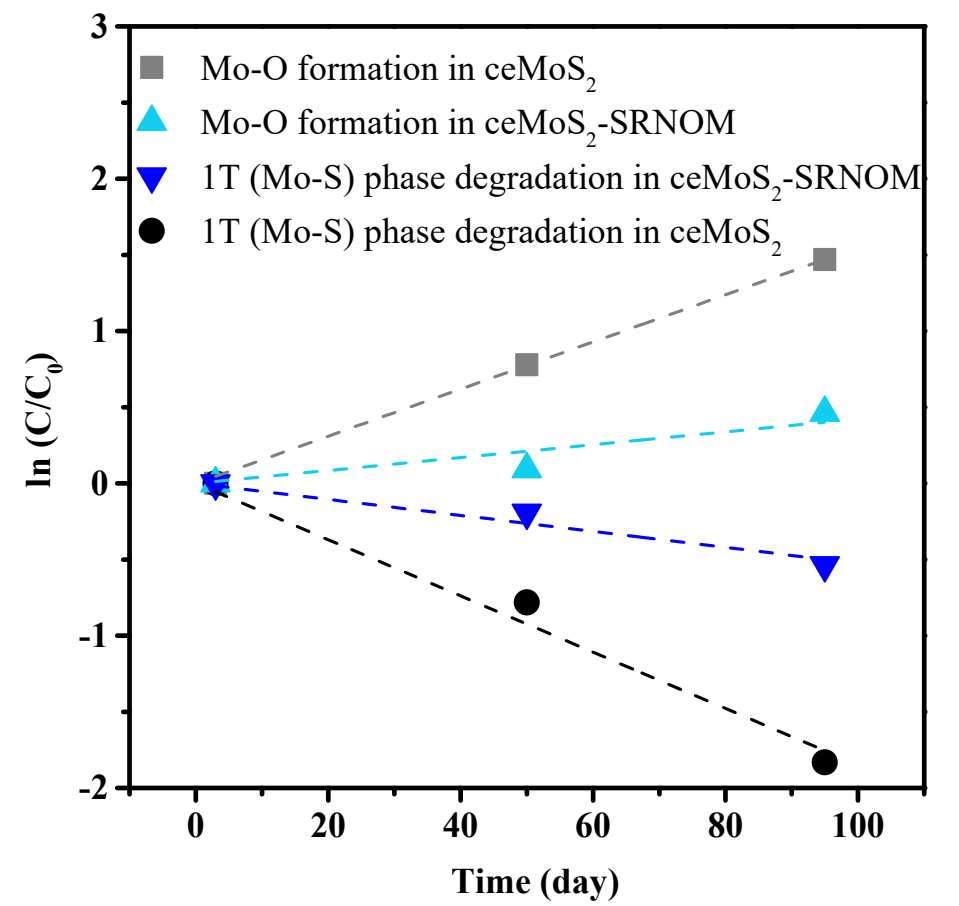

Mo-O formation in ceMoS :

$$
\begin{aligned}
& y=0.0155 x \\
& y=0.0042 x \\
& y=-0.0052 x
\end{aligned}
$$

Mo-O formation in ceMoS 2 -SRNOM:

1T (Mo-S) phase degradation in ceMoS $: \quad y=-0.0185 x$

Figure S9. Kinetic results of Mo-O formation and 1T (Mo-S) phase degradation in $\mathrm{ceMoS}_{2}$ and ceMoS2-SRNOM evaluated by high-resolution XPS spectra (Figure 3). 

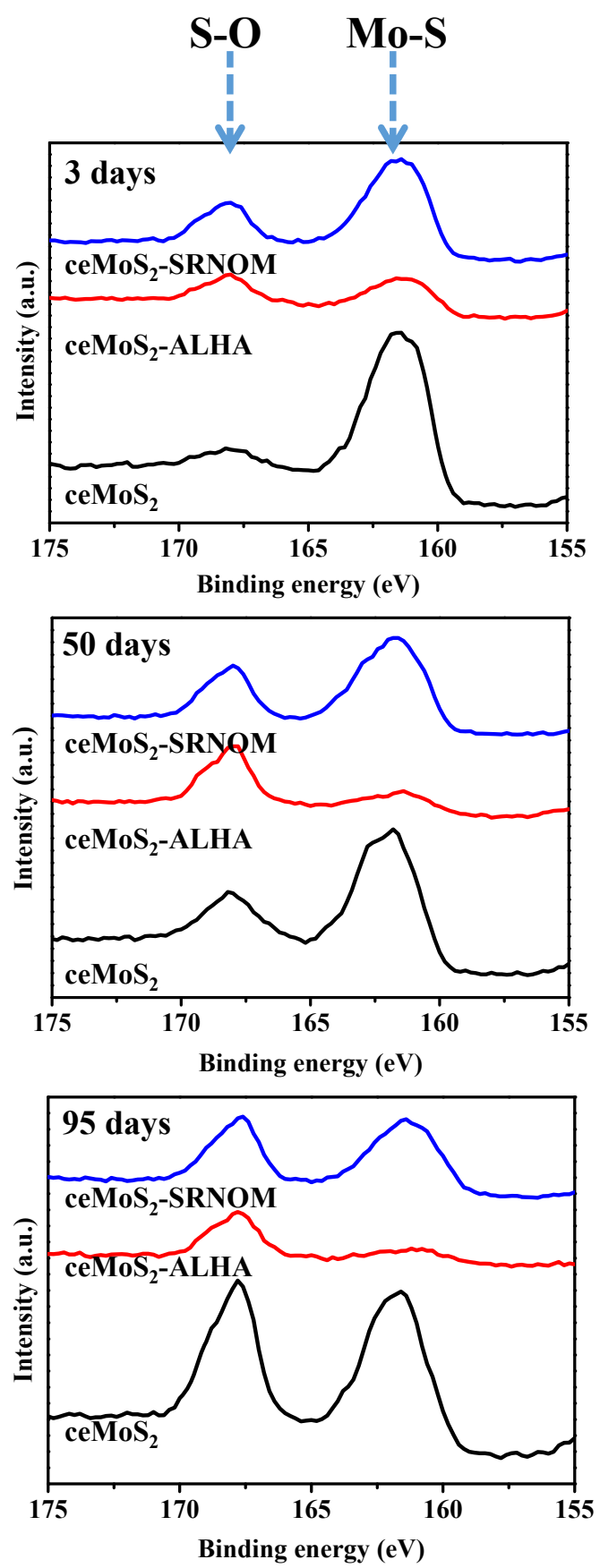

Figure S10. S 2P XPS spectra of ceMoS $2, \mathrm{ceMoS}_{2}-\mathrm{SRNOM}$, and ceMoS 2 -ALHA aged for 3 days, 50 days, and 95 days under dark ambient conditions. 


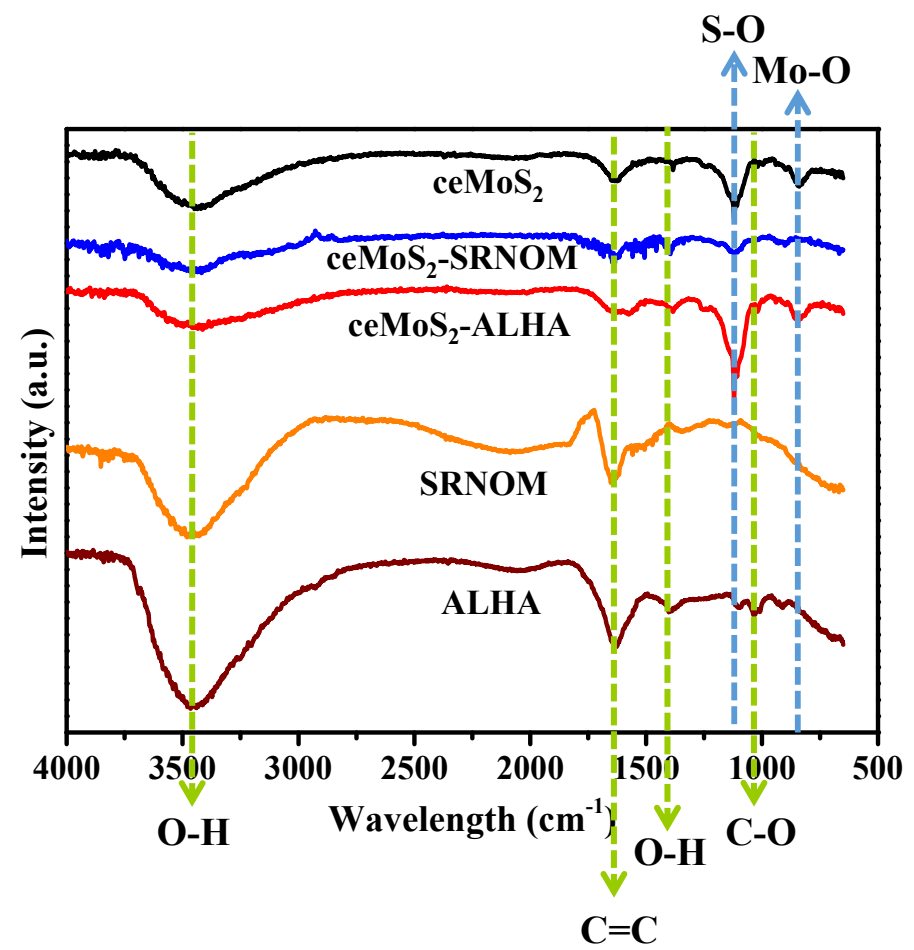

Figure S11. FTIR spectra of 3-day-aged ALHA, SRNOM, ceMoS $2, \mathrm{ceMoS}_{2}-\mathrm{ALHA}$, and ceMoS ${ }_{2-}$ SRNOM. Compared to the spectra of ALHA and SRNOM, Mo-O $\left(\sim 800 \mathrm{~cm}^{-1}\right)^{8}$ and S-O $(\sim 1106$ $\left.\mathrm{cm}^{-1}\right)^{9}$ stretching bonds were observed in ceMoS2-ALHA and ceMoS 2 -SRNOM. Other peaks located at $\sim 1030 \mathrm{~cm}^{-1}$ (C-O stretch), $\sim 1410 \mathrm{~cm}^{-1}$ (O-H bend), $\sim 1630 \mathrm{~cm}^{-1}$ (C=C stretch), and $\sim 3400 \mathrm{~cm}^{-1}$ (O-H stretch ${ }^{10}$ were identified. 

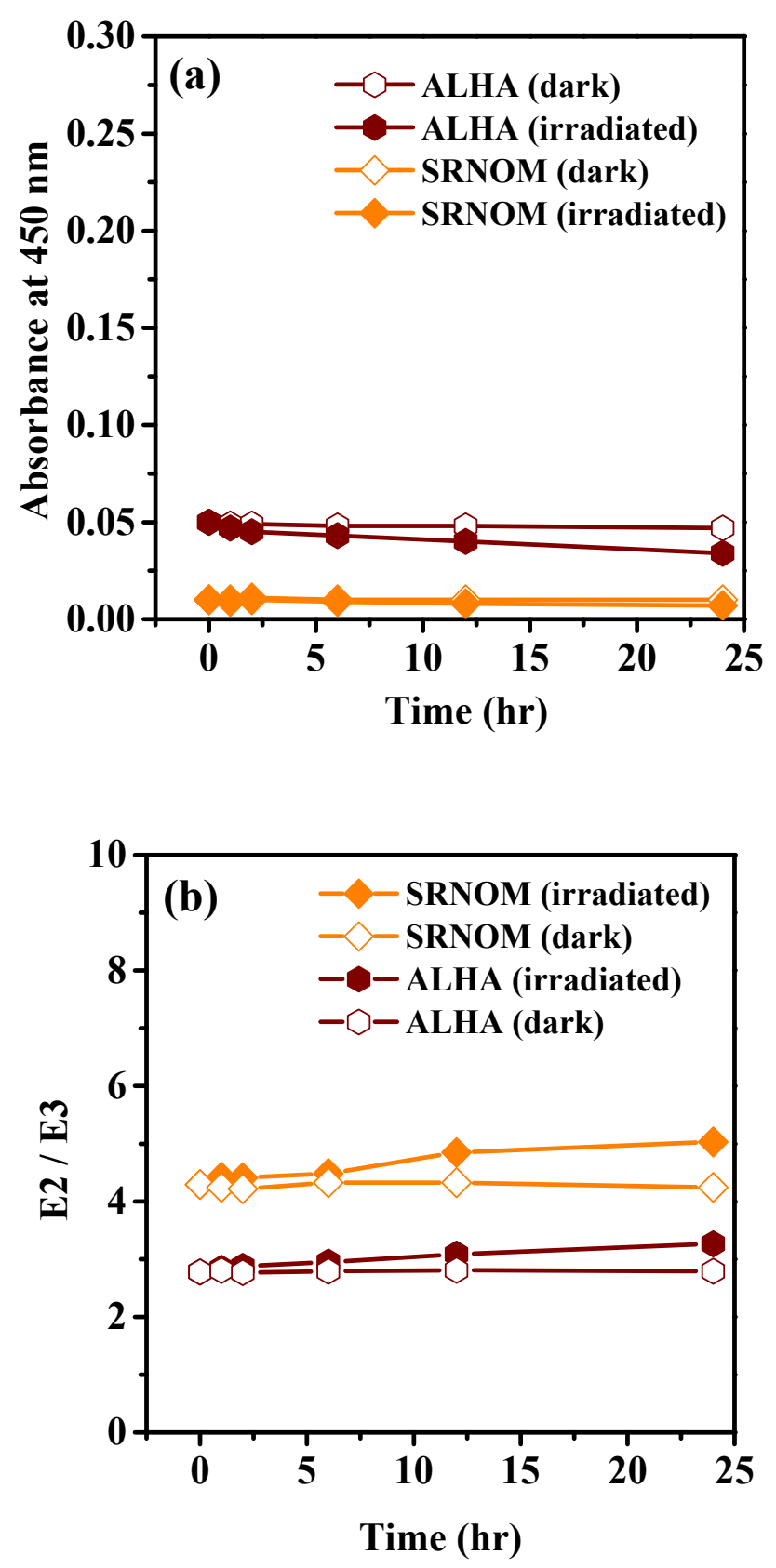

Figure S12. (a) UV-vis absorbance at $450 \mathrm{~nm}$ of ALHA and SRNOM ( $8 \mathrm{mg} / \mathrm{L})$ under sunlight irradiation conditions. (b) The ratio between the absorbance at 254 and $365 \mathrm{~nm}(\mathrm{E} 2 / \mathrm{E} 3)$ is a proxy for the degree of photobleaching of natural organic matter. The stable E2/E3 values indicate negligible photobleaching of SRNOM and ALHA during the 24 h-irradiation. 

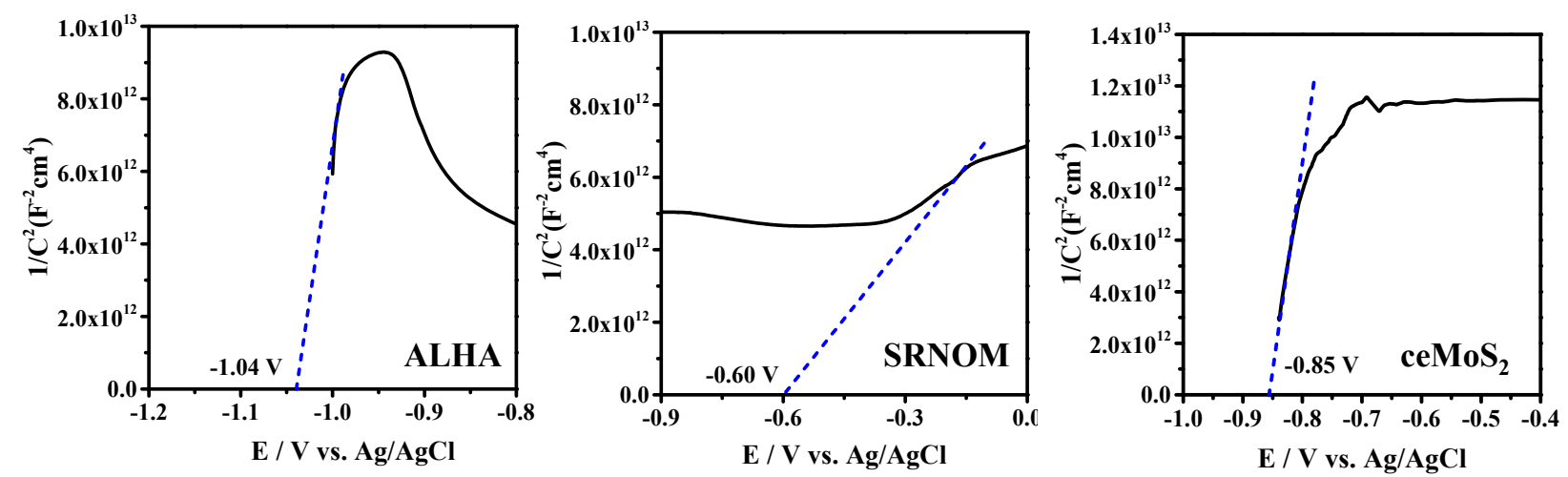

Figure S13. Mott-Schottky plots of ALHA, SRNOM, and ceMoS 2 . Three-electrode configuration comprised of an $\mathrm{Ag} / \mathrm{AgCl}$ electrode as the reference electrode, platinum wire as the counter electrode, and a glassy carbon electrode with samples as the working electrode. A $0.5 \mathrm{M} \mathrm{Na}_{2} \mathrm{SO}_{4}$ aqueous solution was used as the electrolyte. 


\section{References}

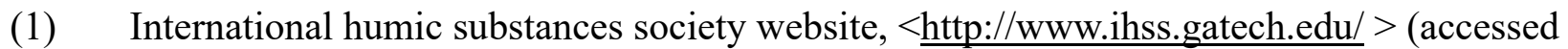
March 2019).

(2) Kang, K.-H.; Shin, H. S.; Park, H. Characterization of humic substances present in landfill leachates with different landfill ages and its implications. Water Research 2002, 36 (16), 4023-4032.

(3) Louie, S. M.; Spielman-Sun, E. R.; Small, M. J.; Tilton, R. D.; Lowry, G. V. Correlation of the physicochemical properties of natural organic matter samples from different sources to their effects on gold nanoparticle aggregation in monovalent electrolyte. Environ. Sci. Technol. 2015, 49 (4), 2188-2198.

(4) Chin, Y. P.; Aiken, G.; Oloughlin, E. Molecular-weight, polydispersity, and spectroscopic properties of aquatic humic substances. Environ. Sci. Technol. 1994, 28 (11), 1853-1858.

(5) Paredes, J. I.; Munuera, J. M.; Villar-Rodil, S.; Guardia, L.; Ayan-Varela, M.; Pagan, A.; Aznar-Cervantes, S. D.; Cenis, J. L.; Martinez-Alonso, A.; Tascon, J. M. D. Impact of covalent functionalization on the aqueous processability, catalytic activity, and biocompatibility of chemically exfoliated $\mathrm{MoS}_{2}$ nanosheets. ACS Appl. Mater. Interfaces 2016, 8 (41), 27974-27986.

(6) Guardia, L.; Paredes, J. I.; Munuera, J. M.; Villar-Rodil, S.; Ayan-Varela, M.; MartinezAlonso, A.; Tascon, J. M. D. Chemically exfoliated $\mathrm{MoS}_{2}$ nanosheets as an efficient catalyst for reduction reactions in the aqueous phase. ACS Appl. Mater. Interfaces 2014, 6 (23), 21702-21710.

(7) Guo, C.; Liu, H.; Wang, J.; Chen, J. Conformational structure of triblock copolymers by FT-Raman and FTIR spectroscopy. Journal of Colloid and Interface Science 1999, 209 (2), 368-373.

(8) Zou, W.; Zhang, X.; Zhou, Q.; Hu, X. Environmental transformations and algal toxicity of single-layer molybdenum disulfide regulated by humic acid. Environ. Sci. Technol. 2018, $52(5), 2638-2648$.

(9) Karade, S. S.; Dubal, D. P.; Sankapal, B. R. MoS2 ultrathin nanoflakes for high performance supercapacitors: Room temperature chemical bath deposition (CBD). RSC Advances 2016, 6 (45), 39159-39165.

(10) Rodríguez, F. J.; Núñez, L. A. Characterization of aquatic humic substances. Water and Environment Journal 2011, 25 (2), 163-170. 